\title{
OBSERVATORIO
}

\section{PÚBLICOS VULNERABLES Y EMPODERAMIENTO DIGITAL: EL RETO DE UNA SOCIEDAD E-INCLUSIVA}

\author{
Vulnerable publics and digital empowerment: The \\ challenge of an e-inclusive society
}

\section{Carmen Fuente-Cobo}

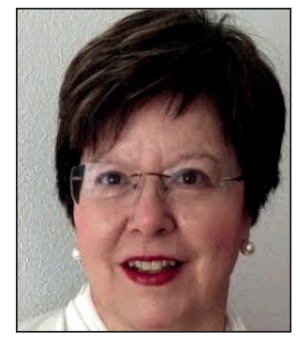

Carmen Fuente-Cobo es profesora titular de periodismo y directora adjunta del área de estudios de comunicación en el Centro Universitario Villanueva (adscrito a la Universidad Complutense de Madrid) desde 2005. Es también titulada por el Programa de Dirección General (PDG) del IESE y cuenta con más de 20 años de experiencia profesional en empresas de contenidos y tecnológicas. Sus líneas de investigación son: ética y profesionalismo periodístico, protección de la infancia en el ámbito audiovisual, y políticas públicas audiovisuales. Es investigadora del Programa de Actividades sobre Vulnerabilidad Digital (Provuldig), financiado por la Comunidad de Madrid (S2015/ HUM-3434).

http://orcid.org/0000-0003-2898-1210

Universidad Complutense de Madrid, Villanueva Centro Universitario, Área de Comunicación Claudio Coello, 11. 28001 Madrid, España cfuentecobo@villanueva.edu

\section{Resumen}

La lucha contra la pobreza y la exclusión social son, con mayor o menor intensidad, una constante en las estrategias europeas de crecimiento económico y generación de empleo, y en los programas marco de investigación vinculados a éstas. En la actualidad los conceptos de exclusión e inclusión incluyen el acceso y uso de tecnologías y redes de información y comunicaciones. Por su relevancia y peso cada vez mayor en las políticas sociales, es preciso avanzar en la definición y aplicación de conceptos como exclusión, vulnerabilidad y empoderamiento en el marco de las sociedades digitales. Para ello, la inversión y dedicación de recursos a la investigación en ciencias sociales y humanidades es una condición fundamental.

\section{Palabras clave}

Vulnerabilidad digital; Empoderamiento; E-inclusión; Sociedad digital; Brecha digital; Horizonte 2020; Plan Estatal de Investigación Científica, Técnica y de Innovación.

\begin{abstract}
The fight against poverty and social exclusion is, to a greater or lesser extent, a constant feature in European strategies for economic growth and employment generation and is featured in related research frameworks andprograms. At present, the concepts of exclusion and inclusion include access to and use of information and communication technologies and networks. Due to its relevance and increasing weight in social policies, it is necessary to make progress in the definition and application of concepts such as exclusion, vulnerability, and empowerment within the framework of digital societies. The investment and dedication of resources to conduct research into the social sciences and humanities is, therefore, a fundamental necessity.
\end{abstract}

\section{Keywords}

Digital vulnerability; Empowerment; E-inclusion; Digital society; Digital divide; Horizon 2020; Spanish State Plan for Scientific, Technical and Innovation Research.

Fuente-Cobo, Carmen (2017). "Públicos vulnerables y empoderamiento digital: el reto de una sociedad e-inclusiva". El profesional de la información, v. 26, n. 1, pp. 5-12.

https://doi.org/10.3145/epi.2017.ene.01 


\section{Introducción}

Hace una década el científico colombiano Manuel Elkin Patarroyo reivindicaba una "ciencia con conciencia social" en la conferencia inaugural de un coloquio internacional celebrado en Granada. Patarroyo, que ha dedicado su vida a la consecución de una vacuna que pueda poner fin a la malaria que todavía aflige a amplias zonas del continente africano, recordaba que la investigación sobre enfermedades que afectan al $90 \%$ de la población mundial recibe únicamente el 10 por ciento de toda la inversión en investigación científica y reclamaba el desarrollo de proyectos científicos centrados en mejorar la vida de las personas.

La llamada de atención del científico colombiano, Premio Príncipe de Asturias de Investigación Científica y Técnica en 1994, no iba dirigida a los investigadores individuales sino más bien a la industria farmacéutica y a las administraciones públicas, que determinan la agenda y prioridades de la inversión en investigación.

La investigación sobre enfermedades que afectan al $90 \%$ de la población mundial recibe únicamente el $10 \%$ de toda la inversión

Dos años antes, con motivo del centenario de la teoría de la relatividad y del cincuentenario del fallecimiento de Albert Einstein, el filósofo Francisco Fernández Buey se refería al genial físico en un artículo que titulaba "ciencia con conciencia" y que aparecía publicado en el mismo año en que lo hacía su libro sobre Einstein, titulado en este caso "Ciencia y conciencia". Posiblemente este segundo título sea más exacto ya que la relación entre ciencia y conciencia de la que habla Fernández Buey al referirse a Einstein no atiende tanto a la unidad de estos dos ámbitos en la actividad investigadora y en el objeto del conocimiento científico, sino que se refiere más bien al perfil personal del científico y a su aprecio por ese otro tipo de conocimiento sapiencial, de tipo humanístico, que le caracterizó como un hombre de mente abierta y juicio independiente $y$ le permitió entenderse y dejar huella en intelectuales de trayectorias y posiciones muy diferentes.

En cualquier caso, hablar de ciencia con conciencia exige tener presente no solamente la figura del científico sino también a las instituciones, organismos y estructuras cuyas decisiones afectan a la selección y orientación de las prioridades de investigación. En el ámbito social, una de las cuestiones recurrentes en las políticas puestas en marcha en la Unión Europea y en España ha sido la lucha contra la pobreza y la exclusión social, realidades que se reconfiguran en el marco de la sociedad digital.

Los objetivos de inclusión social se han intensificado en la estrategia Europa

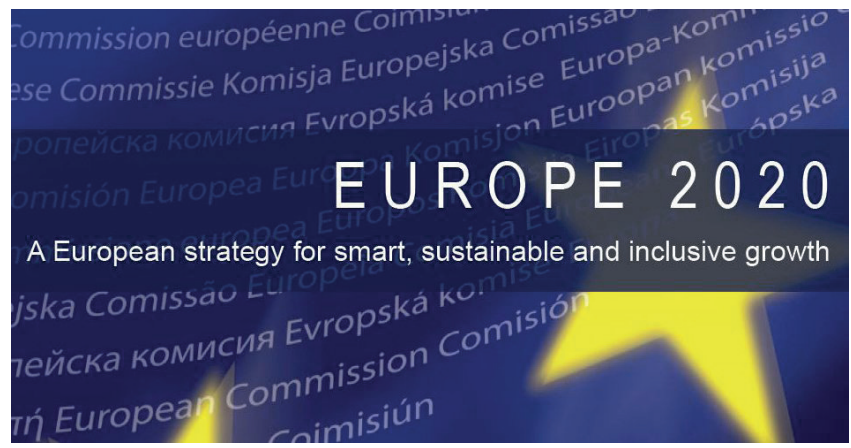

http://ec.europa.eu/europe2020/europe-2020-in-a-nutshell/index_en.htm

2020, aprobada en junio de 2010 y que persigue la consecución de un crecimiento económico basado en el conocimiento y la innovación (crecimiento inteligente), el uso eficaz de los recursos (crecimiento sostenible) y altos niveles de empleo y cohesión social y territorial (crecimiento integrador). La estrategia tiene cinco objetivos: el empleo, la investigación, la innovación, el cambio climático y la energía, y la lucha contra la pobreza. En relación con este último ámbito, el objetivo es reducir el número de personas en situación de pobreza o exclusión en un $25 \%$. En esta situación se encuentran alrededor de 80 millones de personas en Europa.

La estrategia Europa 2020 ha recibido críticas en relación con sus objetivos de lucha contra la pobreza. En particular, se le reprocha que este objetivo no se haya definido de manera más ambiciosa y que pueda quedar diluido dentro del más amplio de crecimiento del empleo, aunque se reconoce como un avance importante el que por primera vez la pobreza y la exclusión social sean una de las prioridades clave de la estrategia europea de crecimiento (Jones, 2010). En la actualidad se asume de manera general que existe una relación entre pobreza y nivel de crecimiento económico y, en consecuencia, la lucha contra la pobreza no debe ser perseguida solamente como un fin en sí mismo, sino también como un medio que incide en el crecimiento económico.

También se ha criticado el indicador elegido para medir la extensión de la pobreza. El indicador que se maneja es

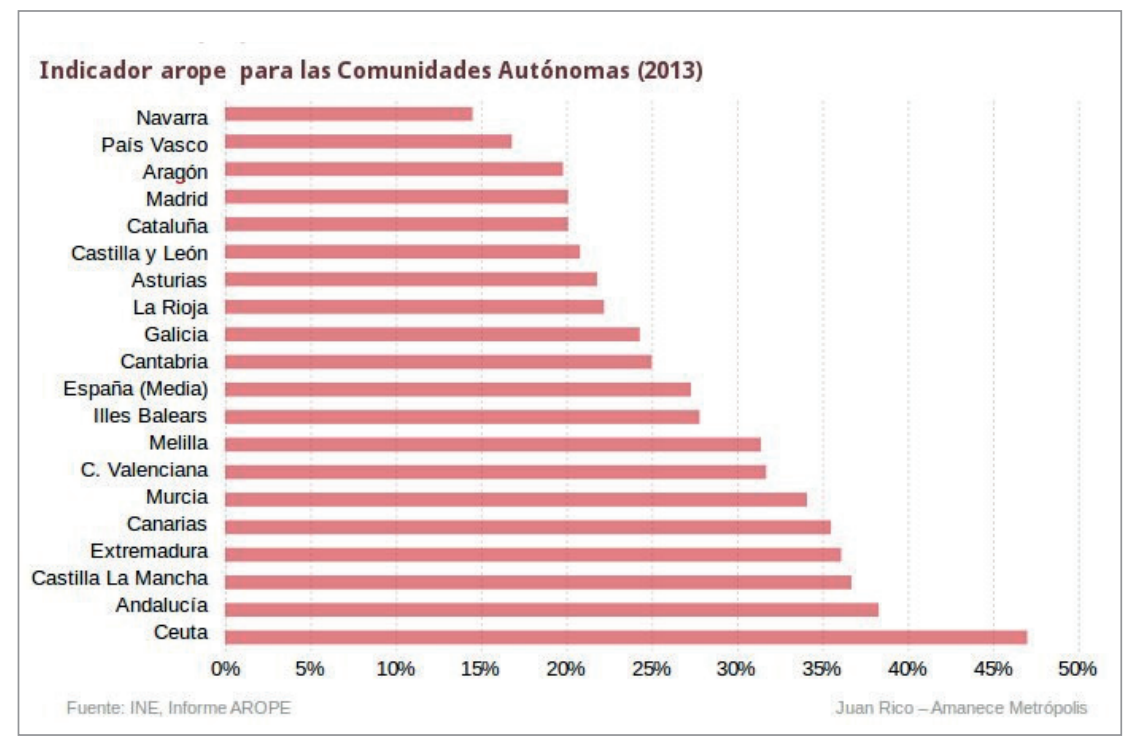

http://amanecemetropolis.net/graficos-arope-crisis-pobreza-y-exclusion-en-espana 
arope (at risk of poverty and/or exclusion), que incluye tres tipos de factores de riesgo, uno de los cuales es la privación material severa. Entran en esta categoría los hogares/ personas que no pueden hacer frente a los gastos de bienes y servicios, entre los cuales se incluye el teléfono (fijo o móvil). Señalan los críticos que el estándar arope necesita ser ampliado con otros indicadores como la educación, las condiciones de vida o el acceso a Internet. De hecho, variables como la tasa de desempleo o el nivel de acceso familiar a internet son muy diferentes en Irlanda o RU frente a España, Grecia o Italia, a pesar de que todos ellos tienen cifras de pobreza similares (Cruz-Morato; García-Lizana, 2015).

\section{El indicador de nivel de pobreza arope incluye tres tipos de factores de riesgo, pero resulta insuficiente}

En relación con estos debates, un repaso de la bibliografía científica producida en los últimos años pone de manifiesto que algunos de los conceptos esenciales que llevamos manejando desde la década pasada y que están plenamente integrados en las políticas y programas de investigación, en la producción científica y en los foros académicos, son todavía imprecisos, tal vez porque actúan como "palabras poderosas" mediante las cuales señalamos aquellos objetivos que percibimos como deseables en el ámbito de las políticas sociales. Es el caso de conceptos como "vulnerabilidad", "exclusión" o "empoderamiento" que, analizados en el marco de la sociedad digital, aparecen vinculados en este monográfico de la revista El profesional de la información.

\section{Exclusión-inclusión y brecha digital}

El recorrido histórico por sus diferentes aproximaciones, desde que fuera popularizado por René Lenoir en su obra Les exclus - Un français sur dix (1974), pone de manifiesto las dificultades que todavía existen para acotar el concepto de exclusión social, que en la actualidad está presente en todas las agendas de política social nacionales e internacionales, como apunta García-Blanco (2016).

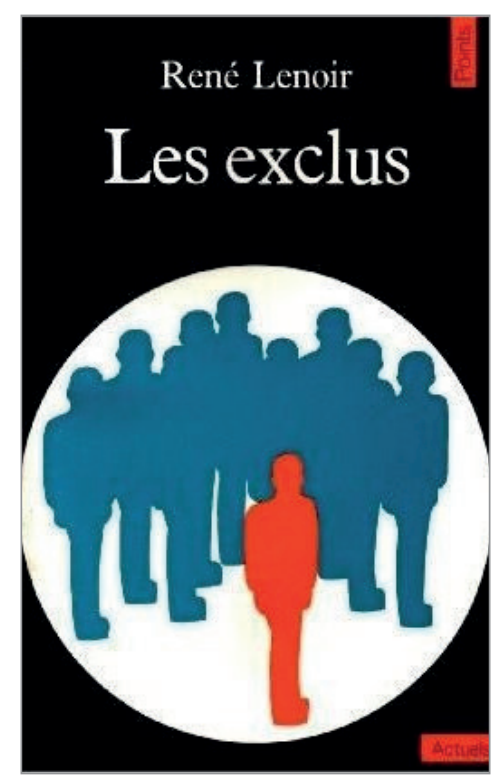

ISBN: 9782020043762

Por lo que se refiere a las estrategias europeas de crecimiento, el concepto de exclusión aparece en los documentos finales del Consejo europeo de Lisboa de marzo de 2000. El objetivo de las estrategias europeas a partir de entonces no es tanto la lucha contra la pobreza cuanto la puesta en marcha de planes para la inclusión social, como señalaba el entonces comisario para Asuntos Económicos y Monetarios de la Unión Europea, Pedro Solbes: "El reto clave es pasar de una agenda centrada en situaciones de exclusión a otra que garantice la inclusión social y la integre en el centro mismo de toda política"1. Todo ello a partir de la constatación de que algunas situaciones de exclusión no tienen como causa directa o única situaciones de carencia económica o material, sino que están relacionadas con privación de derechos humanos y de ciudadanía en un sentido amplio (Subirats, 2004).

En la actualidad, el concepto de exclusión se utiliza con una triple acepción, dependiendo de que las oportunidades de participación del individuo o grupo en la vida social se refieran al ámbito económico, al de la ciudadanía o al relacional. Tezanos define la exclusión social como aquella situación en la que las personas se encuentran fuera de las oportunidades vitales que caracterizan a una ciudadanía social plena. $Y$, aunque él la refiere al momento histórico concreto de finales del siglo XX, el sentido se mantiene para cualquier período, en la medida en que cada tiempo histórico viene acompañado de oportunidades nuevas y diferentes generadas a partir de avances tecnológicos, económicos y sociales. En cualquier caso, las desigualdades sociales que dan lugar a la exclusión se acrecientan en la Sociedad de la Información (Tezanos, 2001, p. 38). Subirats, por su parte, considera la exclusión social como opuesta a la inclusión, entendida ésta como

"un estatuto social sostenido sobre tres pilares: la participación en la producción de valor social dentro o fuera del mercado; la adscripción política y de ciudadanía, y la existencia de contacto con redes sociales y/o familiares" (Subirats, 2005, p. 9).

Algunas situaciones de exclusión no son por carencia económica, sino por la privación de derechos humanos y de ciudadanía

En definitiva, el concepto de exclusión no se entiende ya como una situación vinculada exclusivamente a la pobreza material, sino que se amplía a otro tipo de carencias educativas, relacionales y de oportunidad. Estas carencias implican, por otra parte, que el individuo que las padece se encuentra de una u otra manera limitado en sus derechos sociales, como si fuera un ciudadano de segunda clase. Entre estos derechos sociales, contenidos en el Pacto Internacional de Derechos Económicos, Sociales y Culturales, y a su vez inspirados en la Declaración Universal de Derechos Humanos de 1948 (derecho a la vivienda, a la educación, acceso a la cultura, etc.), no se incluye ciertamente el derecho de acceso a tecnologías de la información y la comunicación. Sin embargo, no cabe entender ya un concepto de inclusión en el que no esté presente la noción de acceso a las herramientas tecnológicas y redes de comunicaciones que constituyen en el mundo presente una de las condiciones básicas para la plena participación social.

La noción de inclusión aparece relacionada, así pues, con la de brecha digital, concepto que comenzó a utilizarse a me- 
diados de los años noventa y que tiene su origen en un informe de la National Telecommunications and Information Agency (NTIA) del Department of Commerce de EUA publicado en julio de 1995, y en el que dicho concepto se utiliza para referirse a las diferencias entre los que tienen acceso a ordenadores e internet, y los que no, los "have not". Por tanto, los excluidos de la sociedad digital son aquellos que reúnen dos condiciones: no tener acceso a la herramienta informática básica, el ordenador; y no tener acceso tampoco a la red, a internet.

Los excluidos de la sociedad digital son los que no tienen ordenador ni acceso a internet o bien no saben utilizarlos adecuadamente

Posteriormente se amplió el concepto: no se trata solamente de tener (equipos, acceso a la red), sino también, y fundamentalmente, de saber utilizar aquello que se tiene. La OCDE definió la brecha digital en 2001 como:

"el espacio que existe entre individuos, hogares, empresas y áreas geográficas con diferentes niveles socioeconómicos, con relación tanto a sus oportunidades de acceder a las tecnologías de la información y la comunicación (TIC) como al uso de internet para una amplia variedad de actividades".

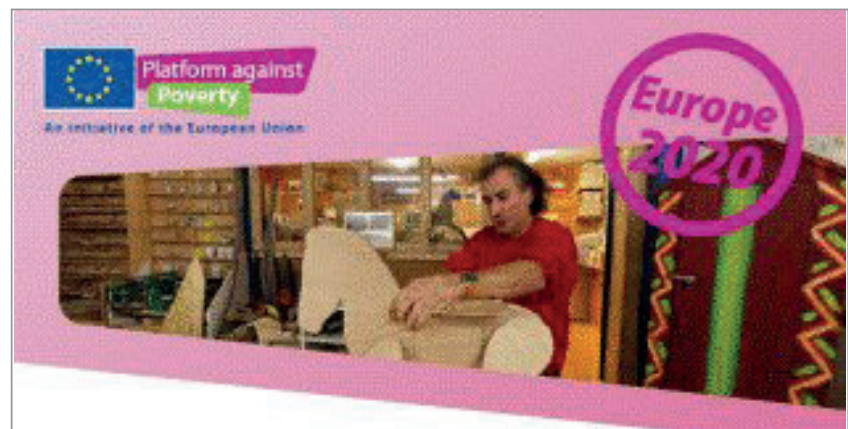

The European Platform against Poverty and Social Exclusion

A European framework for social and ferritarial cohesion

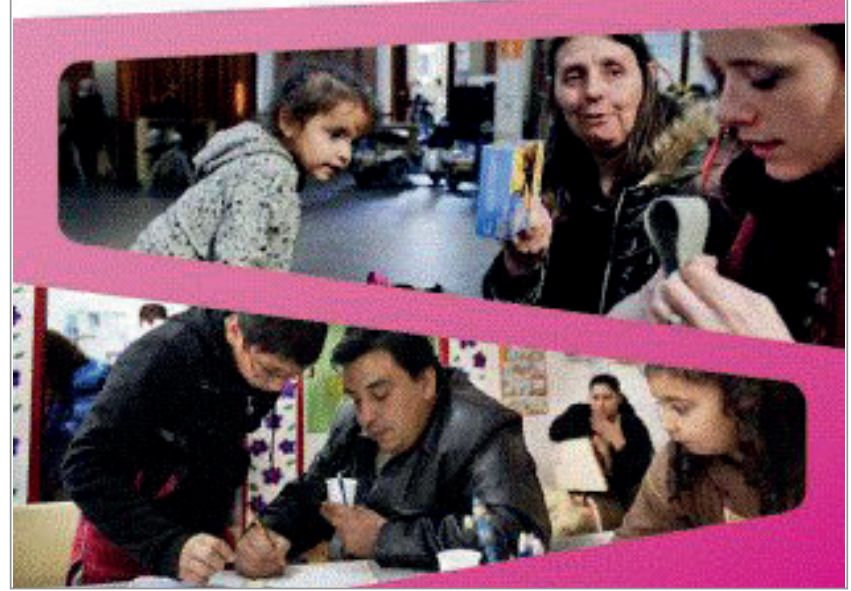

http://ec.europa.eu/social/main.jsp?cat/d=961\&/angld=es
Ballestero (2002) resume las variables que integran el concepto de brecha digital, señalando que ésta se relaciona con cuatro elementos:

- disponibilidad de ordenador u otro elemento en el hogar que permita conexión a internet;

- posibilidad de conectarse y acceder a la red desde el hogar o el trabajo;

- conocimiento de las herramientas básicas para poder acceder y navegar por la Red; y

- capacidad adecuada para poder hacer que la información accesible en la Red pueda ser convertida en 'conocimiento' por el usuario.

\section{Grupos vulnerables}

Entre las muchas cuestiones abiertas se encuentra la identificación de los colectivos vulnerables, es decir, en riesgo de exclusión. La estrategia Europa 2020 se refiere de manera expresa a grupos muy diversos a la hora de señalar posibles áreas de actuación específica. Así, al definir los objetivos de la Plataforma Europea contra la Pobreza y la Exclusión, creada como iniciativa emblemática en el marco de lucha contra la pobreza, apunta como uno de sus objetivos el desarrollo de programas que permitan luchar contra la discriminación laboral y educativo de las "comunidades más desasistidas", entre las que cita los discapacitados, así como el desarrollo de una nueva agenda para la integración de los inmigrantes.

\section{En las políticas puestas en marcha en la Unión Europea y en España ha sido re- currente la lucha contra la pobreza y la exclusión social}

Además, se insta a los Estados miembros a definir y aplicar medidas adaptadas a las circunstancias específicas de grupos que presentan riesgos particulares (por ejemplo, familias monoparentales, ancianos, minorías, pueblo romaní, discapacitados y personas sin hogar).

Subirats (2004) analiza los colectivos incluidos en los Planes Nacionales de Acción para la Inclusión Social en Europa, puestos en marcha en los países en el marco de las propuestas aprobadas en los Consejos Europeos de Lisboa y Feira (marzo y junio de 2000), Nova (noviembre 2000) y Laeken (diciembre 2001). Son la población vulnerable, tal como ésta es identificada en los programas de acción social europeos. Elegidos por 10 o más países se encuentran los siguientes grupos de riesgo: discapacitados, menores, inmigrantes y asilados, personas sin hogar, mujeres, jóvenes, y personas drogodependientes. A éstos se añaden los elegidos por cinco o más países: familias, ancianos, exreclusos, personas con problemas psíquicos, alcohólicos y minorías étnicas. Y finalmente, los elegidos por menos de 5 países: pobres, parados, prostitutas, viajeros, inmigrantes retornados, y analfabetos.

Exclusión y vulnerabilidad son estados interrelacionados, en la medida en que podemos entender la exclusión como un proceso (Castel, 2004) con tres zonas, desde cada una de las cuales se puede acceder a la otra: 
- la zona de integración (en la que los elementos clave son empleo y relaciones sociales estables);

- la zona de vulnerabilidad (precariedad laboral e inestabilidad en las relaciones sociales); y

- la zona de exclusión social (Castel, 1994). A ellas Tezanos (2001) incorpora la zona asistencial, entre la vulnerabilidad y la exclusión, en la que se incluyen los destinatarios de subsidios sociales. Hablamos por tanto de vulnerabilidad para referirnos a las personas y grupos sociales en riesgo de tránsito hacia zonas de exclusión.

La vulnerabilidad, en un sentido amplio, se predica no solamente de aquellos que forman parte de colectivos en situación de riesgo de exclusión, sino que se utiliza también para referirse a individuos y grupos susceptibles de sufrir daño en su acceso y exposición a los medios y servicios de la sociedad de la información. Entran en este apartado, de manera muy especial, los niños, para los que se vienen definiendo políticas específicas casi desde que la televisión se convirtió en un medio masivo.

\section{Son vulnerables las personas y grupos sociales en riesgo de tránsito hacia zo- nas de exclusión}

\section{Empoderamiento, ¿para qué?}

Recordaba Alex Grijelmo recientemente en su columna semanal sobre el uso del lenguaje (El país, 20 nov. 2016), el itinerario seguido por el verbo "empoderar" y su acción "empoderamiento", desde que en 1925 fueran introducidos en el Diccionario de la Real Academia como sinónimos de "apoderar" y "apoderamiento", hasta su desaparición en la edición de 2001 y su reincorporación en la de 2014. La reciente recuperación de estas palabras viene de la mano de su uso en lengua inglesa, de donde se ha tomado el sentido ampliado con el que ahora se aplica. Aunque mantiene como primera acepción la sinonimia con el verbo apoderar, en la segunda acepción incluida por la $R A E$ empoderar significa "hacer poderoso o fuerte a un individuo o grupo social desfavorecido".

En la actualidad el concepto de "empoderamiento" se aplica a tantos y tan diferentes tipos de situaciones y grupos sociales y tipos de intervención, que difícilmente podemos concluir que para que se dé el efecto de empoderamiento el punto de partida debe ser el de una situación de desventaja económica. Es decir, el concepto tiende a aplicarse de manera generosa y amplia en el sentido que también le daba la RAE en la edición de 2005 de su Diccionario Panhispánico de Dudas, de "conceder poder [a un colectivo desfavorecido socioeconómicamente] para que, mediante su autogestión, mejore sus condiciones de vida". En cualquier caso, el poder para el que se empodera es entendido como una propiedad dinámica susceptible de crecer o disminuir, por lo que junto con la investigación centrada en el empoderamiento de grupos e individuos en diferentes ámbitos, encontramos también resultados de trabajos que describen efectos de "desempoderamiento" en la sociedad de la información (Mariën; Prodnik, 2014).

Una de las perspectivas más fructíferas desde las que se ha abordado esta cuestión del empoderamiento en las dos últimas décadas es la relativa a la alfabetización digital. La investigación centrada inicialmente en el ámbito de lo estrictamente mediático y, más en concreto, en el de los medios audiovisuales (Gozálvez-Pérez; Contreras-Pulido, 2014), ha tenido la virtud de situar en primer plano la relevancia del concepto de competencia y la compresión del mismo como una realidad que integra dimensiones no solamente relacionadas con el acceso y uso de medios y recursos, sino también con la capacidad para descodificarlos críticamente e interactuar con ellos, siguiendo a Ferrés y Piscitelli (2012).

La alfabetización digital es entendida también como un proceso que permite diseñar estrategias de e-inclusión específicamente enfocadas a colectivos vulnerables como es la tercera edad, para la que el objetivo que se define es recuperar su capacidad de interacción social y su participación cívica, un objetivo que exige a su vez tener en cuenta las circunstancias personales y sociales de cada individuo (Abad, 2014).

Otra de las perspectivas desde las que es abordado el empoderamiento digital es el de las oportunidades profesionales en el campo del periodismo y la información de interés público generadas por el fenómeno "open data", aunque se advierte que no puede establecerse una rela- 
ción directa entre más datos abiertos y empoderamiento, cambio social o niveles mayores de democracia (Gertrudis; Gértrudix, 2016).

Los menores, como colectivo especialmente vulnerable, son también objeto de la atención investigadora a través de la educación mediática y digital (Tejedor; Pulido, 2012) que busca minimizar los riesgos de un uso inadecuado de medios y redes y a la exposición que los menores tienen en éstas ${ }^{2}$. Este empoderamiento se extiende a la familia, ámbito para el que se plantean iniciativas de alfabetización digital de reforzamiento de la mediación parental, al tiempo que se reclaman políticas públicas y privadas de refuerzo de los sistemas de información y selección de contenidos por parte de padres y educadores.

También los jóvenes son objeto de estrategias de empoderamiento, por ejemplo las que se definen en el entorno audiovisual, entendiendo aquí por empoderamiento la capacitación para usar tecnologías de producción para que los jóvenes puedan hacer oír su voz (Aguaded; Carrero, 2013).

El concepto de empoderamiento se vincula también a la participación ciudadana a través de internet (Cáceres; Brändle; Ruiz-San-Román, 2015). En este sentido, es entendido como e-facilitación, es decir, como aprovechamiento de las posibilidades que ofrece la Red, para visibilizar iniciativas, reivindicaciones y causas que de otra manera no tendrían oportunidad de ser oídas. Se trata no sólo del ciberactivismo sino también de la cibersolidaridad y de las denominadas tecnologías sociales (Saorín; GómezHernández, 2014).

La administración española parece haber cuidado la proporcionalidad con las CSH a la hora de realizar las adjudicaciones

\section{El papel de las ciencias sociales y humanidades en la agenda de I+D+i}

Desde 1982 las actividades de investigación e innovación en la UE se coordinan por medio de programas marco. El que está actualmente en vigor es el Programa Marco de Innovación e Investigación (2014-2020) conocido como Horizonte 2020, cuyo objetivo central es la construcción de una sociedad y una economía basadas en el conocimiento y la innovación en toda la Unión, por medio de tres pilares: ciencia excelente, liderazgo industrial, y atención a los retos de la sociedad.

Son siete los grandes retos sociales que el programa europeo identifica para la Unión Europea en la actualidad:

1. Salud, cambio demográfico y bienestar.

2. Seguridad alimentaria, agricultura y silvicultura sostenibles, investigación marina, marítima y de aguas interiores y bioeconomía.

3. Energía segura, limpia y eficiente.

4. Transporte inteligente, ecológico e integrado.

5. Acción por el clima, medio ambiente, eficiencia de los recursos y materias primas.
6. Europa por un mundo cambiante-Sociedades inclusivas, innovadoras y reflexivas.

7. Sociedades seguras. Proteger la libertad y la seguridad de Europa y sus ciudadanos.

Respecto al reto número 6 relativo a la consecución de sociedades inclusivas, innovadores y reflexivas, los indicadores de partida ponen de manifiesto hasta qué punto el concepto de inclusión incorpora ya la alfabetización digital como condición (el $25 \%$ de los ciudadanos de la UE, se indica, no poseen alfabetización digital). Los grandes temas que se plantean en relación con este reto de la inclusión (participación, migración, integración, cambio demográfico, derechos humanos, y justicia mundial) demuestran su complejidad y la necesidad de ser abordados desde una perspectiva multidisciplinar.

Esta necesidad de incorporar enfoques multidisciplinares está en la base de la decisión de integrar las ciencias sociales y las humanidades de manera transversal a partir de la convocatoria de 2014 del programa Horizonte 2020. Se entiende, en este sentido, que la contribución de campos de investigación y actividad como la sociología, la economía, la psicología, las ciencias políticas, la historia, las denominadas ciencias de la cultura, el derecho y la ética, es necesaria "para generar nuevo conocimiento, apoyar la elaboración de políticas basadas en evidencias, desarrollar competencias clave y producir soluciones interdisciplinares tanto a cuestiones tecnológicas como sociales" ${ }^{\prime 3}$.

Los resultados de la ronda 2014/15 reflejan en qué medida este objetivo de integrar ciencias sociales y humanidades (CSH) en la investigación sobre los grandes retos sociales a los que se enfrenta Europa en esta década, está siendo alcanzado por el programa Horizonte 2020.

- El presupuesto total del programa para 2014 fue de 4.000 millones de euros.

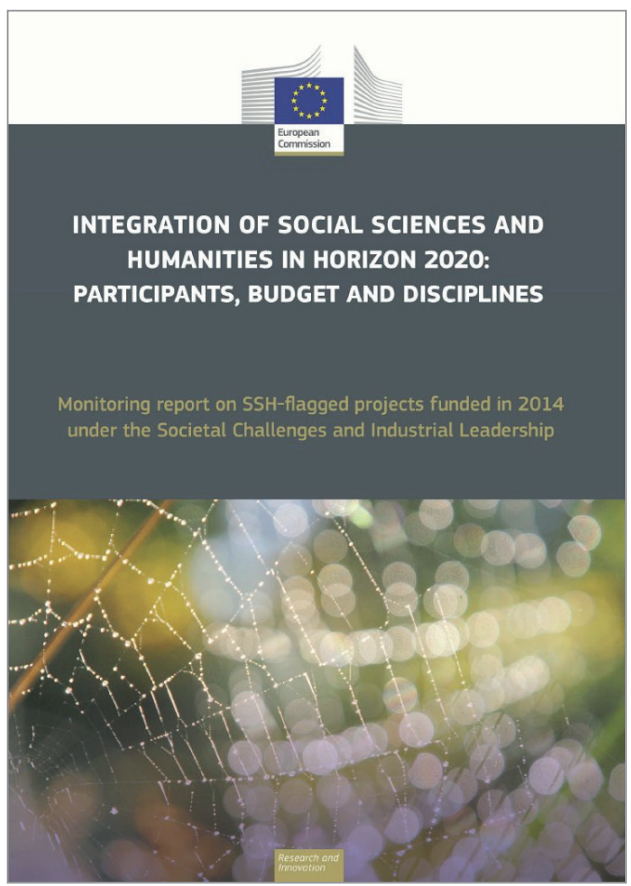

https://ec.europa.eu/programmes/horizon2020/en/news/integration-socialsciences-and-humanities-horizon-2020-participants-budget-and-disciplines 
- El 37\% de todos los temas lanzados en esta convocatoria llevaban la etiqueta de $\mathrm{CSH}$, lo que en términos prácticos implica que tenían como objetivo incorporar las CSH como expertise plenamente integrado dentro de cada proyecto.

- El presupuesto asignado a socios (partners) de perfil CSH para todos los proyectos/temas presentados en relación con los siete retos del programa $\mathrm{H} 2020$ fue del $6 \%$.

- El presupuesto asignado al reto 6 (sociedades inclusivas) fue de 114 millones de euros (2,8\% del total). De éstos, 70 millones de euros $(61,4 \%)$ fueron destinados a socios de perfil CSH).

- Sólo 77 de los 308 proyectos financiados bajo la etiqueta de proyectos con temática CSH fueron coordinados por un socio con perfil $\mathrm{CSH}$.

- Respecto a la representación por disciplinas, los socios procedentes de ciencias económicas, negocios y marketing fueron mayoría ( $53 \%$ de los proyectos con socios de $\mathrm{CSH}$ ), seguidos por los expertos de ciencias políticas, administración pública y derecho (38\%) y muy por delante de psicología (19\%), sociología (17\%), humanidades y artes (9\%), demografía y geografía (6\%), antropología y etnología (3\%), educación y comunicación ( $2 \%)$, e historia (2\%).

En España, el marco rector de la política en I+D+i es la Estrategia Española de Ciencia y Tecnología y de Innovación 2013-2010, cuyo instrumento principal es el Plan Estatal de Investigación Científica, Técnica y de Innovación 2013-2016, que coincide e integra, a su vez, objetivos del programa europeo Horizonte 2020.

El Plan Estatal está integrado por cuatro Programas Estatales que a su vez se subdividen en subprogramas. Así, el Programa Estatal de $1+D+i$ orientado a los Retos de la Sociedad se subdivide en 7 subprogramas, uno de los cuales tiene su foco puesto en los cambios e innovaciones sociales, e incluye entre los temas de atención prioritaria cuestiones relacionadas con desigualdad, exclusión y pobreza.

Un análisis rápido de los proyectos aprobados en la convocatoria de 2016 pone de manifiesto que la administración española parece haber cuidado la proporcionalidad a la hora de realizar las adjudicaciones, ya que los proyectos marcados en algunos de los ocho campos incluidos por el Ministerio de Economía y Competitividad en el área temática de gestión de ciencias sociales y humanidades representan aproximadamente la cuarta parte del total de proyectos adjudicados ${ }^{4}$. Si atendemos a las disciplinas representadas, de los 324 proyectos aprobados en esta convocatoria, el mayor número correspondió a los de derecho (63), seguidos de las ciencias sociales (61), economía (54), historia y arte (38), psicología (38), filología y filosofía (24), educación (24), deporte (13) y estudios feministas (9).

Obviamente, el análisis debe entrar en la cuantía y alcance de estos proyectos para poder generar conclusiones válidas acerca de la importancia real de las ciencias sociales y las humanidades en la generación de conocimiento sobre los grandes retos sociales y, sobre todo, como guía y soporte para las políticas sociales.

Los artículos que integran el presente número de El profesional de la información, algunos de los cuales están basa-

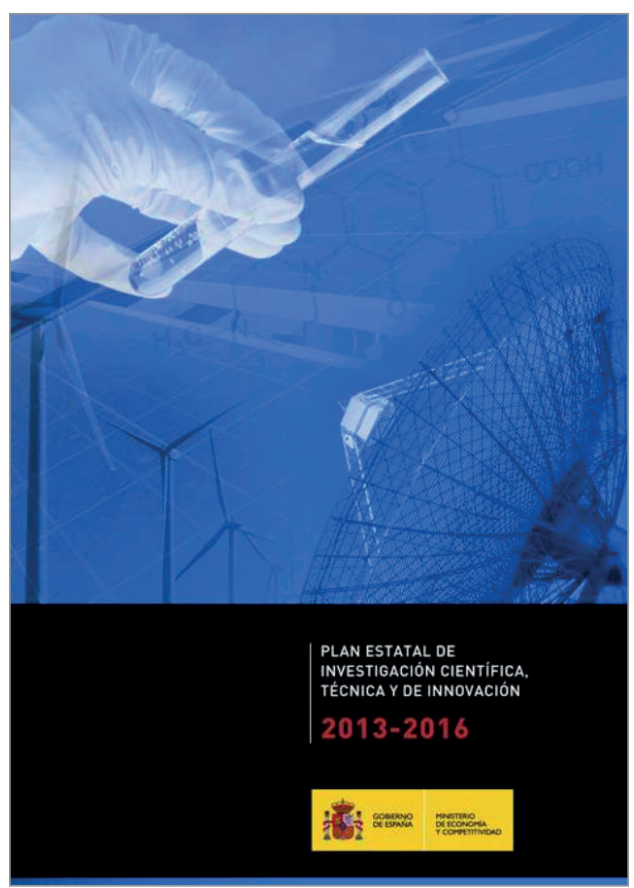

http://www.idi.mineco.gob.es/stfIs/MICINN/Investigacion/FICHEROS/ Plan_Estatal_Inves_cientifica_tecnica_innovacion.pdf

dos en proyectos de investigación aprobados en convocatorias competitivas como las comentadas, son un reflejo de la variedad de colectivos vulnerables que se identifican en la actualidad y de la diversidad de enfoques y estrategias definidos para su empoderamiento.

\section{Notas}

1. Pedro Solbes, prólogo a la obra de Fernando Ballestero, La brecha digital (ver referencias).

2. Destaca en esta línea el trabajo del equipo de investigación constituido desde hace varios años en la Universidad del País Vasco, vinculado al proyecto europeo EU Kids online, y su informe más reciente sobre menores e Internet, que acaba de ser publicado en diciembre de 2016. (Ver: Garmendia-Larrañaga et al., Net children go mobile. Riesgos y oportunidades en internet y dispositivos móviles entre menores españoles (2010-2015). Informe final. Universidad del País Vasco - Red.es. Proyecto cofinanciado por el Programa Safer Internet de la Comisión Europea, el Ministerio de Economía y Competitividad y la entidad pública Red.es.

3. European Commission. Integration of Social Sciences and Humanities in Horizon 2020: Participants, budget and disciplines. Monitoring report on SSH-flagged projects funded in 2014 under the Societal Challenges and Industrial Leadership.

\section{4. http://www.idi.mineco.gob.es/portal/site/MICINN}

\section{Bibliografía}

Abad, Leopoldo (2014). "Diseño de programas de e-inclusión para alfabetización mediática de personas mayores". Comunicar, n. 42, pp. 173-180.

https://doi.org/10.3916/C42-2014-17

Aguaded-Gómez, José-Ignacio; Sánchez-Carrero, Jacqueline (2013). "El empoderamiento digital de niños y jóvenes a través de la producción audiovisual". adComunica. Revista 
de estrategias, tendencias e innovación en comunicación, n. 5, pp. 175-196.

https://doi.org/10.6035/2174-0992.2013.5.11

Ballestero, Fernando (2002). La brecha digital. El riesgo de exclusión en la Sociedad de la Información. Fundación Retevisión. ISBN: 9788493154295

Cáceres-Zapatero, María-Dolores; Brändle, Gaspar; RuizSan-Román, José-Antonio (2015). "Hacia la construcción de una ciudadanía digital. Nuevos modelos de participación y empoderamiento a través de Internet". Prisma social, n. 15, pp. 643-683.

https://goo.gl/dw7Lml

Castel, Robert (1994). "La dynamique des processus de marginalisation: de la vulnerabilité à la désaffiliation". Cahiers de recherche sociologique, n. 22, pp. 11-27. http://dx.doi.org/10.7202/1002206ar

Castel, Robert (2004). "Los riesgos de exclusión social en un contexto de incertidumbre". Revista internacional de sociología, v. 72, pp. 15-24.

https://doi.org/10.3989/ris.2013.03.18

Comisión Europea (2014). Horizon 2020 en breve. El programa Marco de Investigación e Innovación de la Unión Europea. https://goo.gl/H5fXVT

Comisión Europea. Europe 2020. Una estrategia para un crecimiento inteligente, sostenible e integrados. COM (2010) 2020 final.

https://goo.gl/9pcelC

Cruz-Morato, Marco-Antonio; García-Lizana, Antonio (2015). "Pobreza, exclusión social y crecimiento económico en la Unión Europea". XII Jornadas internacionales de política económica. Toledo, mayo 2015.

http://www.riuma.una.es/xmlui/handle/10630/9857

European Commission (2014). Integration of Social Sciences and Humanities in Horizon 2020: Participants, budget and disciplines. Monitoring report on SSH-flagged projects funded in 2014 under the Societal Challenges and Industrial Leadership. https://goo.gl/gHPtNH

Fernández-Buey, Francisco (2005). "Ciencia con conciencia". Quark, n. 36, pp. 91-95.

http//quark.prbb.org/36/default.htm

Ferrés, Joan; Piscitelli, Alejandro (2012). "La competencia mediática: propuesta articulada de dimensiones e indicadores". Comunicar, n. 38, pp. 75-82.

https://doi.org/10.3916/C38-2012-02-08

García-Blanco, José-María (2016). “Los problemas teóricos y metodológicos del concepto de exclusión social. Una visión neofuncionalista". RIS. Revista internacional de sociología, v. 74, n. 2.

https://doi.org/10.3989/ris.2016.74.2.029

Garmendia-Larrañaga, Maialen; Jiménez-Iglesias, Estefanía; Casado, Miguel-Ángel; Mascheroni, Giovanna (2016). Net children go mobile. Riesgos y oportunidades en internet y dispositivos móviles entre menores españoles (20102015). Informe final.
https://goo.gl/M1PBE8

Gertrudis-Casado, María-Carmen; Gértrudix-Barrio, Manuel; Álvarez-García, Sergio (2016). "Competencias informativas profesionales y datos abiertos. Retos para el empoderamiento ciudadano y el cambio social". Comunicar, n. 47, pp. 39-47.

https://doi.org/10.3916/C47-2016-04

Gozálvez-Pérez, Vicent; Contreras-Pulido, Paloma (2014). "Empoderar a la ciudadanía mediática desde la educomunicación". Comunicar, n. 42, pp. 129-136. https://doi.org/10.3916/C42-2014-12

Jones, Sian (2010). "La Estrategia Europa 2020, ¿influirá en la pobreza?". Revista de la Red Europea de Lucha contra la Pobreza y la Exclusión, n. 133, pp. 6-7.

http://www.eapn.es/ARCHIVO/documentos/recursos/3/873_ Mag133_ES_web.pdf

Mariën, Ilse; Prodnik, Jernej A. (2014) “Digital inclusión and user (dis)empowerment: A critical perspective. info, v. 16, n. 6, pp. 35-47.

https://doi.org/10.1108/info-07-2014-0030

Ministerio de Economía y Competitividad. Estrategia Española de Ciencia y Tecnología y de Innovación 2013-2016. https://goo.gl/qnzkHV

Ministerio de Economía y Competitividad. Plan Estatal de Investigación Científica, Técnica y de Innovación 2013-2016. https://goo.gl/2IL6xa

National Telecommunications and Information Administration (NTIA), US Dept. of Commerce (1995). Falling through the Net: A survey of the 'have nots' in rural and urban America. https://www.ntia.doc.gov/ntiahome/fallingthru.html

OECD (2001). Understanding the digital divide. https://www.oecd.org/sti/1888451.pdf

Saorín, Tomás; Gómez-Hernández, José-Antonio (2014). "Alfabetizar en tecnologías sociales para la vida diaria y el empoderamiento". Anuario ThinkEPI, v. 8, pp. 342-348.

http://www.thinkepi.net/alfabetizar-en-tecnologias-socialespara-la-vida-diaria-y-el-empoderamiento

Subirats, Joan (dir.) (2004). Pobresa i exclusió social. Una anàlisi de la realitat espanyola i europea. Fundació La Caixa. Col·lecció Estudis socials, n. 16.

http://www.didacqueralt.com/pobresa-cat.pdf

Subirats, Joan (dir.) (2005). Perfils d'exclusió social urbana a Catalunya. Una aproximació qualitativa. Institut de Govern i Polítiques Públiques. Universitat Autònoma de Barcelona.

https://ddd.uab.cat/pub/llibres/2005/37/exclusiosocial_ a2005.pdf

Tejedor, Santiago; Pulido, Cristina (2012). "Retos y riesgos del uso de Internet por parte de los menores, ¿Cómo empoderarlos?". Comunicar, n. 39, pp. 65-72.

https://doi.org/10.3916/C39-2012-02-06

Tezanos, José-Félix (2001). La sociedad dividida. Estructura de clases y desigualdades en las sociedades tecnológicas. Madrid: Biblioteca Nueva. ISBN: 9788470308932 